Original Paper

\title{
Validation of Capillary Zone Electrophoretic Method for Evaluating Monoclonal Antibodies and Antibody-Drug Conjugates
}

\author{
Kei KUBOTA $^{* 1,2}$, Naoki KOBAYASHI², Masayuki YABUTA³ ${ }^{3}$, Motomu OHARA², \\ Toyohiro NAITO ${ }^{1}$, Takuya KUBO ${ }^{1}$, Koji OTSUKA ${ }^{1}$ \\ ${ }^{1}$ Graduate School of Engineering, Kyoto University, Katsura, Nishikyo-ku, Kyoto 615-8510, Japan \\ ${ }^{2}$ Analytical and Quality Evaluation Research Laboratories, Daiichi Sankyo Co., Ltd., 1-12-1 Shinomiya,
}

Hiratsuka 254-0014, Japan

${ }^{3}$ Biologics Technology Research Laboratories, Daiichi Sankyo Co., Ltd., 2716-1 Aza Kurakake, Oaza Akaiwa,Chiyoda-machi,

Oura 370-0503, Japan

\begin{abstract}
Based on capillary zone electrophoresis (CZE), we validated a method of confirming the identity and purity of the separated charge variants of monoclonal antibodies (mAbs) and antibody-drug conjugates (ADCs). The validation includes the evaluation of the specificity, linearity, quantitation limit, precision (repeatability and intermediate precision), accuracy, range, and robustness. The method is applicable to the majority of mAbs and ADCs (with $\mathrm{p} I$ from 7 to 9 and a drug to antibody ratio up to 8), requiring no modification to the method conditions. The proposed CZE method showed reproducible separation profiles, while cation exchange chromatography (CEX) showed low reproducibility and deficient separation profiles due to an undesirable interaction between the separation column and the low molecular weight drugs combined in the ADCs. Since CZE is able to minimize this undesirable interaction during the separation, it proved to be a useful separation methodology for evaluating charge variants of ADCs. The validation of CZE for assessing ADCs was successfully demonstrated for the first time, and show that CZE is suitable for the separation method for detecting the charge heterogeneity of ADCs.
\end{abstract}

Keywords: Capillary zone electrophoresis; Charge heterogeneity; Monoclonal antibody; Antibody-drug conjugate; Validation

\section{Introduction}

Monoclonal antibodies (mAbs) and antibody-drug conjugates (ADCs) have emerged as promising classes of therapeutics in the biopharmaceutical industry because of their advantages of having highly specific targeting towards a wider range of indications. More than $40 \mathrm{mAbs}$ have been approved for use in indications such as cancer and inflammatory diseases [1-5]. ADCs combine the potency of cytotoxic drugs with the high specificity of mAbs and have become increasingly important as new targeted therapies in oncology. The primary sites used for protein-directed conjugation are the amino groups of lysine residues or the sulfhydryl groups of inter-chain cysteine residues. Two ADCs have been approved by the FDA for the treatment of late-stage metastatic breast cancer and relapsed Hodgkin's lymphoma [6-13].

Charge heterogeneity is a very important characteristic in mAbs and ADCs because it relates to their quality, stability, and efficacy. Every product has its own primary structure, thus showing its specific $\mathrm{p} I$. Changes of charge profile suggests several post translational modifications, such as $C$-terminal variants (lysine truncation [14] and proline amidation [15]), deamidation [16,17], glycation [18,19], and pyroglutamic acid cyclization [20,21]. These modifications are generated during production and storage of products [22]. Some modifications of amino acids

Received: 21 July 2016

Accepted: 24 August 2016

J-STAGE Advance Published: 1 September 2016

DOI: 10.15583 jpchrom.2016.011
Tel: +81-463-31-6912; Fax: +81-463-38-4049

E-mail: kubota.kei.c7@daiichisankyo.co.jp 
located in complementarity determining regions reduce the binding activity of antigens [23]. Therefore, an evaluation of charge heterogeneity is significant to the identification of the products and the monitoring of the quality of the products based on their charge profiles.

To evaluate charge variants of $\mathrm{mAbs}$, cation exchange chromatography (CEX) [24-30], isoelectrophoretic focusing (IEF) [31], capillary IEF (cIEF) [32-34] and imaged cIEF (icIEF) [35-38] are usually used. However, these methods require many experimental optimizations, which take a long time for measurement, and the obtained data is usually less quantitative and less reproducible. Capillary zone electrophoresis (CZE) has become increasingly accepted as an attractive alternative to IEF and CEX for assessment of charge heterogeneity of mAbs, because CZE allows simpler to conduct experiments, faster to obtain results, and high reproducibility [39-47].

The charge profiles of ADCs are changeable during production and storage, because the antibody itself suffers from degradation, leading to charge heterogeneity as described above. Therefore, it is desirable to monitor charge variants of ADCs to assure their quality is maintained. However, charge-based development methods and validation for ADCs has so far rarely been reported [48]. Charge variant analysis of ADCs is challenging due to (1) the hydrophobicity of low-molecular weight drugs, which causes an undesirable hydrophobic interaction during the analysis (i.e., with CEX columns), (2) the complexity caused by the charge heterogeneity of naked antibodies and low-molecular weight drugs themselves, and (3) the variation of drug distribution. CZE require no denaturants or solid-phase interfaces as separation media, enabling the inherent heterogeneity of ADCs to be analyzed at close to their native states. Therefore, CZE is suitable for separation method for detecting the charge heterogeneity of ADCs.

In this study, the method validation of CZE was conducted for use with an identity and purity test for mAbs and ADCs. The validation items consisted of specificity, linearity, quantitation limit, precision (repeatability and intermediate precision), accuracy, range and robustness. We believe that the validation of CZE for assessing ADCs was successfully demonstrated for the first time, and CZE is applicable not only for mAbs but also ADCs.

\section{Experimental}

\subsection{Materials and reagents}

The mAbs and ADCs were all manufactured at Daiichi Sankyo Co., Ltd. (Tokyo, Japan). 6-Aminocaproic acid (EACA), hydroxypropyl methyl cellulose (HPMC) and triethylenetetramine (TETA) were purchased from Sigma-Aldrich (Saint Louis, MO, USA). Acetic acid, 0.1 $\mathrm{mol} / \mathrm{L}$ hydrochloric acid, sodium chloride, $2 \mathrm{~mol} / \mathrm{L}$ sodium hydroxide and urea were purchased from Wako Pure
Chemical Industries (Osaka, Japan). A neutral capillary was purchased from Beckman Coulter, Inc. (Brea, CA, USA). An analytical ProPac WCX-10 column $(250 \times 4$ $\mathrm{mm}$ i.d.) was purchased from Thermo Fisher Scientific, Inc. (Waltham, MA, USA). 2-Morpholinoethanesulfonic acid, monohydrate (MES) was purchased from Dojindo Laboratories (Kumamoto, Japan).

\section{2. $C E X$}

The CEX analysis was conducted on a Shimadzu Prominence LC20 HPLC system equipped with a UV detector. A ProPac WCX-10 column and mobile phase A (20 mmol/L phosphate buffer $\mathrm{pH}$ 6.6) and mobile phase $\mathrm{B}$ ( $0.5 \mathrm{~mol} / \mathrm{L}$ sodium chloride in mobile phase $\mathrm{A})$ were used with a gradient of $0 \%$ to $40 \%$ mobile phase B for 39 min at a flow rate of $0.5 \mathrm{~mL} / \mathrm{min}$. The sample was not diluted with mobile phase $\mathrm{A}$, and $5 \mu \mathrm{L}$ of the sample was injected into the column. The column temperature was $40^{\circ} \mathrm{C}$ with detection at $280 \mathrm{~nm}$.

\section{3. $C Z E$}

The CZE analysis was performed on a Beckman PA800 plus system equipped with a UV detector. The capillary was thermostated at $25^{\circ} \mathrm{C}$, and detection was performed at $214 \mathrm{~nm}$. The capillary $(50 \mu \mathrm{m} \mathrm{id}$, total length $50 \mathrm{~cm}$, effective length $40 \mathrm{~cm}$ ) was used with a constant voltage of $30 \mathrm{kV}$ for all analyses. Before each sample injection, the capillary was rinsed with $0.1 \mathrm{~mol} / \mathrm{L}$ hydrochloric acid, running buffer of $0.05 \% \mathrm{HPMC}, 380 \mathrm{mM}$ EACA, and 1.9 mM TETA, pH 5.7. Samples were diluted with water (for $\mathrm{mAbs}$ ) or their formulation buffer (for ADCs) to $2 \mathrm{mg} / \mathrm{mL}$ and injected at $0.5 \mathrm{psi}$ for $10 \mathrm{~s}$.

\subsection{Method validation}

The validations consisted of the specificity (measurement of formulation buffer, degraded sample, and other samples), linearity, quantitation limit, precision (repeatability and intermediate precision), accuracy, range, and robustness. As model samples, two different mAbs and two different ADCs were selected: $\mathrm{mAb}-\mathrm{A}$, of which theoretical $\mathrm{p} I$ was 9.0; mAb-B, of which theoretical $\mathrm{p} I$ was 7.4 ; ADC-C, of which drug antibody ratio (DAR) was relatively low (DAR 4); and ADC-D, of which DAR was relatively high (DAR 8). For the specificity test, samples were incubated in chambers for a specific period of time to prepare degraded samples. Detailed information for each test is described below.

\subsubsection{Specificity}

The formulation buffer, degraded samples, and other $\mathrm{mAb}$ samples were measured to confirm the specificity of the method.

\subsubsection{Linearity}


Seven test samples, of which concentrations were 1, 5, 10, $25,50,100$, and $200 \%$ of the target concentration (2 $\mathrm{mg} / \mathrm{mL}$ ), were measured. The correlation coefficients and Area/Conc. (\%) for main and total peak areas were calculated as a function of sample concentrations.

\subsubsection{Quantitation limit}

The concentration providing $\mathrm{S} / \mathrm{N} \geq 10$ and Area/Conc. (\%) of the main peak within $\pm 30 \%$ of that of the $100 \%$ concentration was determined as the limit of quantitation (LOQ) of this method. The relative standard deviation (RSD) of the main peak area in this concentration was calculated. The measurement was repeated six times.

\subsubsection{Precision; Repeatability and Intermediate precision}

Repeatability was evaluated by six consecutive analysis of the samples. The \%RSD of the peak area of the main peak in the target concentration was calculated. In addition, the RSD of the migration time of the main peak was calculated.

Intermediate precision was demonstrated following with the design of the experiment summarized in Table 1. Experimental days, analysts, capillary lots and instruments were set as the experimental factors. The \% RSD of the peak area of the main peak in the target concentration was calculated.

Table 1. Design of experiment for intermediate precision.

\begin{tabular}{ccccc}
\hline Day & Analyst & $\begin{array}{c}\text { Capillary } \\
\text { lot }\end{array}$ & Instrument & Repetition \\
\hline 1 & A & X & $\alpha$ & 2 \\
2 & B & Y & $\alpha$ & 2 \\
3 & B & Z & $\alpha$ & 2 \\
4 & B & X & $\beta$ & 2 \\
5 & A & Y & $\beta$ & 2 \\
6 & A & Z & $\beta$ & 2 \\
\hline
\end{tabular}

\subsubsection{Accuracy}

Accuracy was evaluated on the basis of the specificity, linearity and precision studies.

\subsubsection{Range}

Range was determined from the basis of the linearity, precision and accuracy studies.

\subsubsection{Robustness (Running buffer components)}

The samples at the target concentration were measured by using running buffers with different $\mathrm{pH}$ and HPMC concentrations $(0.05 \%$ HPMC $\mathrm{pH} 5.7 \pm 0.1,0.05 \% \pm$ $0.005 \%$ HPMC pH 5.7).

\section{Results and discussion}

\subsection{CEX method development for ADCs}

The conjugation of drugs to mAbs increases the structural complexity of a product, which triggers the need for improved separation methods. However, charge-based methods for evaluating ADCs have rarely been reported. First, we demonstrated a CEX method for charge variant evaluation of ADC-D (which had a DAR 8). Fig. 1 shows an example of CEX chromatograms of ADC-D, indicating low separation efficiency and low reproducibility. The separation of ADC charge variants was not improved by changing sample preparation (with or without dilution by its formulation buffer), mobile phase $\mathrm{pH}$ or gradient programs. However, when the naked antibody (without the low-molecular weight drug of ADC-D) and other mAbs were analyzed, the method showed robust and reproducible results. These results indicate that the low robustness of the CEX method for the ADC was caused by some ADC specific characteristics, such as the hydrophobicity of a low-molecular weight drug. The validation of the CEX method for assessing ADCs was difficult as long as we solved the undesirable interaction of the low-molecular drug with the CEX column and confirmed reproducible results. Therefore, we concluded that the CEX method was not suitable for evaluating charge variants of ADCs qualitatively, and developed another alternative method, $\mathrm{CZE}$, to obtain reproducible separation profile of ADCs.

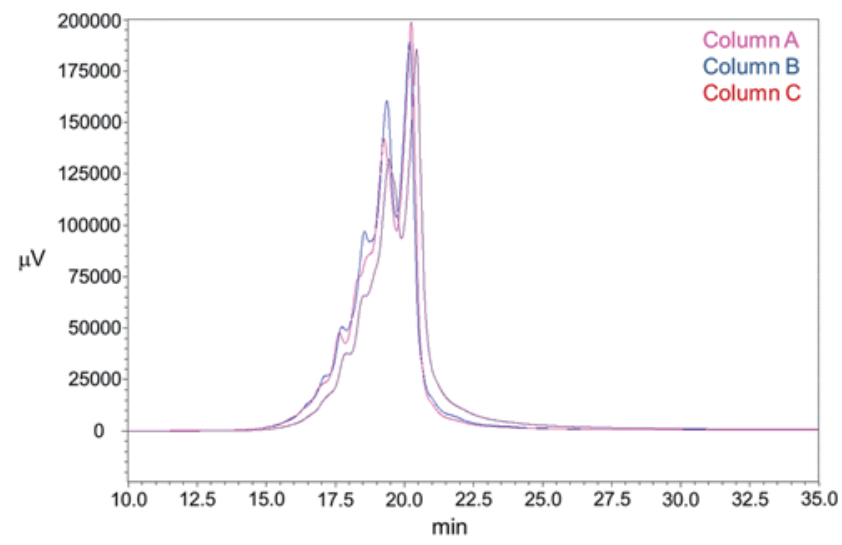

Fig. 1. Chromatograms of ADC-D using three lots of CEX columns. Analytical conditions: analytical column, ProPac WCX-10 column $(250 \times 4 \mathrm{~mm}$ i.d. $)$; mobile phase A, 20 $\mathrm{mmol} / \mathrm{L}$ phosphate buffer $\mathrm{pH} 6.6$, mobile phase $\mathrm{B}, 0.5 \mathrm{~mol} / \mathrm{L}$ sodium chloride in mobile phase A; flow rate, $0.5 \mathrm{~mL} / \mathrm{min}$; gradient program, $0 \%$ to $40 \%$ mobile phase B for $39 \mathrm{~min}$; column temperature, $40^{\circ} \mathrm{C}$; detection, $280 \mathrm{~nm}$; injection, $5 \mu \mathrm{L}$.

\subsection{Summary of method validation of CZE for $m A b s$ and $A D C s$}

A CZE method for assessing mAbs and ADCs was demonstrated and validated. The principle of the method was the same as the method reported by $\mathrm{He}$ et al [35]. Briefly, we modified some experimental conditions, such as the use of a neutral capillary to reduce sample adsorption to the capillary inner-wall and a sample preparation to ensure a lower LOQ and longer sample solution stability. The samples were diluted with water (for mAbs) or their formulation buffer (for ADCs) to the $2 \mathrm{mg} / \mathrm{mL}$ sample 
concentration as a standard condition. Two mAb samples, mAb-A (pI 9) and mAb-B ( 1 I 7), and two ADC samples, ADC-C (having a lower drug to antibody ratio [DAR] 4) and ADC-D (having a higher DAR 8), were used to ensure the wide range of the method applicability. The validation of the method, including the specificity, linearity, quantitation limit, precision, accuracy and robustness, was conducted. The summary of the validation results is listed in Table 2. The method was applicable to more than 10 products, including ADCs without any modification, and showed specific migration times and separation profiles. Therefore, the CZE method is useful to identify and quantitate the charge variants of mAbs and ADCs in the manufacturing process and quality evaluation of biopharmaceuticals. Detailed results are described in following sections.

Table 2. Summary of CZE validation results.

\begin{tabular}{|c|c|c|c|c|}
\hline Parameter & $\mathrm{mAb}-\mathrm{A}$ & $\mathrm{mAb}-\mathrm{B}$ & $\mathrm{ADC}-\mathrm{C}$ & ADC-D \\
\hline \multicolumn{5}{|l|}{ Specificity } \\
\hline Formulation buffer & \multicolumn{4}{|c|}{ Separated from formulation buffer components. } \\
\hline Degradation sample & \multicolumn{4}{|c|}{ Detected the degraded changes. } \\
\hline Other mAbs/ADCs & \multicolumn{4}{|c|}{ Observed specific migration times and separation profiles. } \\
\hline \multicolumn{5}{|l|}{ Linearity } \\
\hline peak area & $\mathrm{R}=1.000$ & $\mathrm{R}=1.000$ & $\mathrm{R}=0.999$ & $\mathrm{R}=0.999$ \\
\hline Area/Conc. $(\%)$ & $92-107 \%$ & $82-102 \%$ & $93-123 \%$ & $92-121 \%$ \\
\hline LOQ & $1.0 \%$ & $1.0 \%$ & $1.0 \%$ & $1.0 \%$ \\
\hline RSD of peak area & $8.7 \%$ & $7.2 \%$ & $7.2 \%$ & $3.9 \%$ \\
\hline \multicolumn{5}{|l|}{ Precision; } \\
\hline \multicolumn{5}{|l|}{ Repeatability } \\
\hline RSD of peak area $\%$ & $0.6 \%$ & $0.8 \%$ & $2.5 \%$ & $0.8 \%$ \\
\hline $\begin{array}{l}\text { RSD of migration } \\
\text { time }\end{array}$ & $0.4 \%$ & $0.1 \%$ & $0.1 \%$ & $0.1 \%$ \\
\hline \multicolumn{5}{|l|}{ Precision; } \\
\hline \multicolumn{5}{|l|}{ Intermediate precision } \\
\hline RSD of peak area $\%$ & $1.4 \%$ & $1.4 \%$ & $1.2 \%$ & $2.3 \%$ \\
\hline Accuracy & Pass & Pass & Pass & Pass \\
\hline Range & $1-200 \%$ & $1-200 \%$ & $1-200 \%$ & $1-200 \%$ \\
\hline $\begin{array}{l}\text { Robustness } \\
\text { (Running buffer } \\
\text { components) }\end{array}$ & \multicolumn{4}{|c|}{$\begin{array}{l}\text { Robust for } \mathrm{pH} \text { changes (pH } 5.7 \pm 0.1) \text { and } \\
\text { HPMC concentrations }(0.05 \% \pm 0.005 \% \text { HPMC) }\end{array}$} \\
\hline
\end{tabular}

\subsection{Specificity}

Electropherograms of the samples and their formulation buffers are shown in Fig. 2. No interference peaks were observed from the formulation buffer around sample derived peaks. One peak was detected around $6 \mathrm{~min}$ in mAb-B, ADC-C and ADC-D. The peak was identified as L-histidine, which has a UV absorbance at $214 \mathrm{~nm}$ and is positively charged at $\mathrm{pH}$ 5.7. The peak was sufficiently separated from the samples and thus, had a negligible impact on the evaluation of the purity of the samples. Therefore, the method specifically detect the sample and (a)

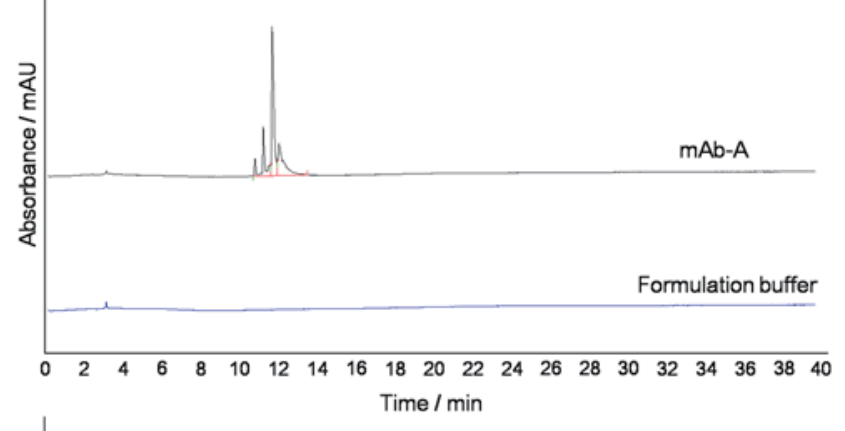

(b)

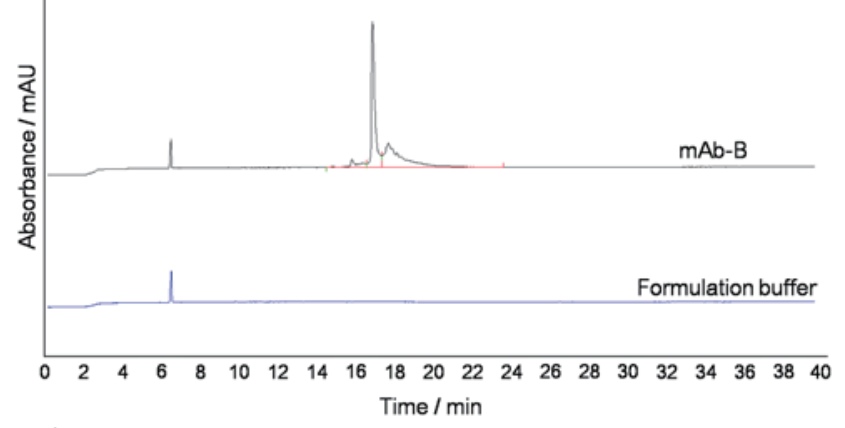

(c)

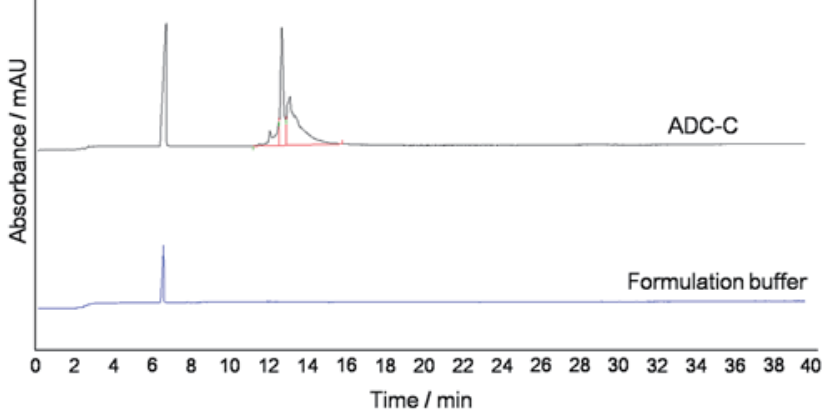

(d)

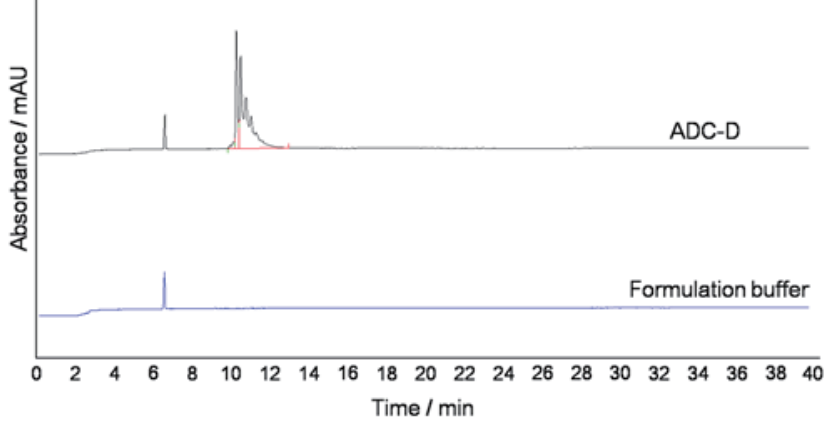

Fig. 2. Electropherograms of (a) mAb-A, (b) mAb-B, (c) ADC-C, (d) ADC-D and their formulation buffers. The formulation buffers of mAb-B, ADC-C and ADC-D contain L-histidine as a buffering salt. Analytical conditions: neutral capillary, i.d./o.d. 50/360 $\mu \mathrm{m} / \mu \mathrm{m}$ and effective/total length of $40 / 50 \mathrm{~cm} / \mathrm{cm}$; separation voltage, $+30 \mathrm{kV}$; detection, $214 \mathrm{~nm}$; capillary temperature, $25^{\circ} \mathrm{C}$; sample storage temperature, $15^{\circ} \mathrm{C}$; injection, 0.5 psi for 10 s; 0.05\% HPMC, 380 mM EACA, 1.9 mM TETA, pH 5.7; $2.0 \mathrm{mg} / \mathrm{mL}$ samples. 
quantify its charge variants.

Electropherograms of the initial and degraded samples are shown in Fig. 3. Changes in the electropherograms between the initial and degraded samples were observed. Especially, acidic peak group (APG) migrating after the main peak was increased and the main peak was decreased in the degradation samples. It is well known that thermal degradation causes deamidation and leads to the increase of APG, which has been confirmed in IEF and CEX $[16,22,29]$. The increase of the APG in CZE agreed well with these results. Therefore, CZE demonstrated its ability to monitor the degradation of samples, and will be useful for stability testing of biopharmaceuticals.

Electropherograms of 11 samples ( 9 mAbs and 2 ADCs) are shown in Fig. 4. Changes in the electropherograms were observed where migration times and peak profiles were apparently different. Therefore, it was confirmed that the method is able to differentiate each sample specifically. The analyzed samples have a wide range of the $\mathrm{p} I$ value (from 7 to 9) and variation of drug distribution (DAR 0 to 8), which are common characteristics in biopharmaceuticals. Therefore, in regards to the specificity, the results indicate that the CZE method can fully cover almost all common variations of biopharmaceutical candidates and be applicable as a universal method for the use with identity tests.

\subsection{Linearity}

The correlation coefficients of all samples were more than 0.999. Area/Conc. (\%) at each concentration of all samples was in the range of $82 \%$ to $123 \%$. Therefore, the linearity in the range of $1 \%$ to $200 \%$ of the target concentration (2 $\mathrm{mg} / \mathrm{mL})$ was confirmed with good recovery.

\subsection{Quantitation limit}

The S/Ns of mAb-A, mAb-B, ADC-C and ADC-D obtained from the sample solutions having $1 \%$ of the target concentration were 13.0, 11.6, 10.1 and 13.9, respectively. The result of the repeatability using the concentration of the sample solutions showed that the RSD of the main peak area was less than $8.7 \%$. Considering the content of the main peak of mAb-A, mAb-B, ADC-C and ADC-D, quantitation limits of the peak areas were determined to be $0.5 \%, 0.5 \%, 0.3 \%$ and $0.4 \%$, respectively. These are sensitive enough to assess the purity of charge variants.

\subsection{Precision (Repeatability and Intermediate precision)}

Fig. 5. shows the results of mAb-A and ADC-C as examples of a repeatability evaluation. The $\% \mathrm{RSD}$ of the peak area of the main peak was less than $2.5 \%$ and the RSD of the migration time of the main peak was less than $0.4 \%$. The $95 \%$ confidence interval of the SD of the migration time indicated that the 0.1 min difference in the migration (a)

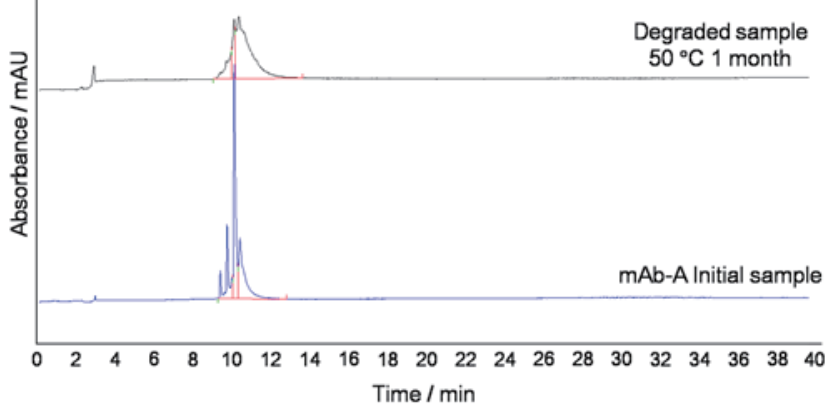

(b)

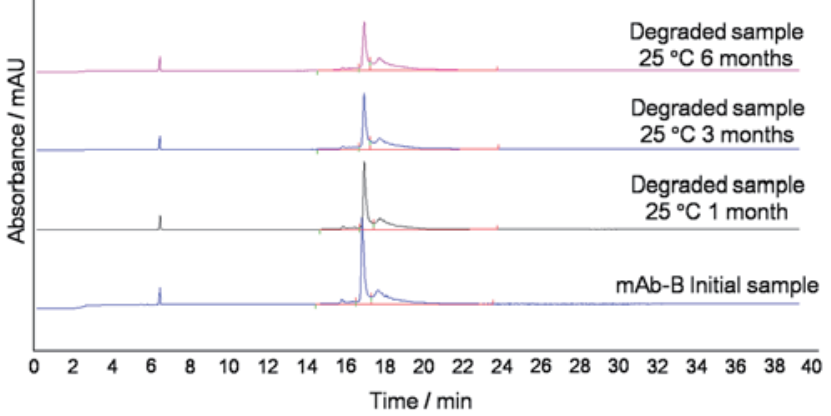

(c)

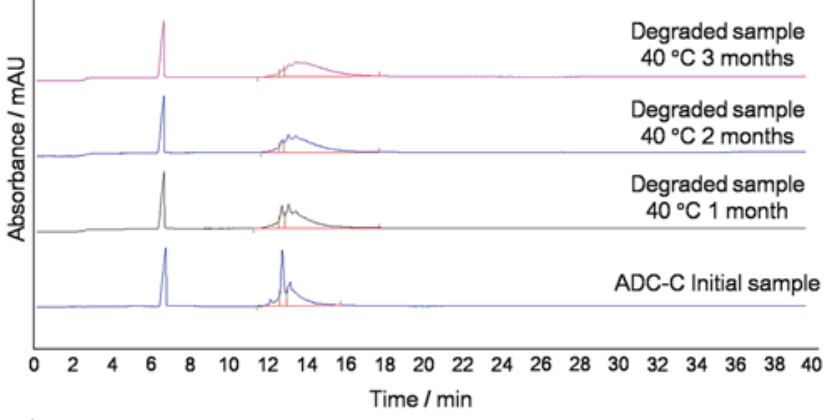

(d)

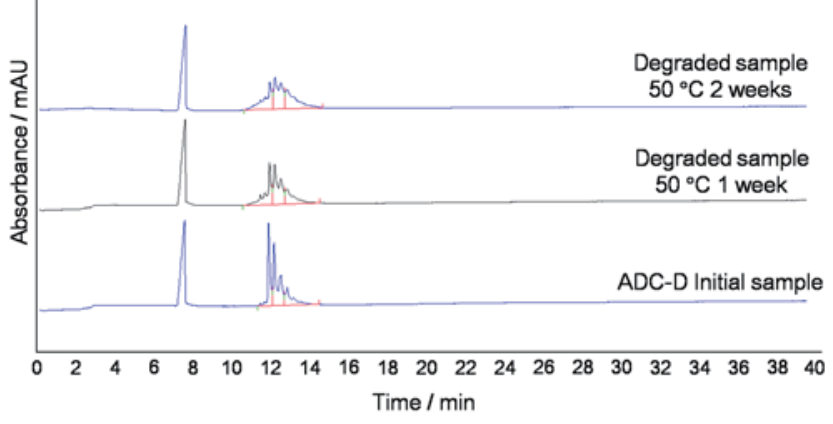

Fig. 3. Electropherograms of (a) mAb-A, (b) mAb-B, (c) ADC-C, (d) ADC-D and their degraded samples. Experimental conditions were the same as those in Fig. 2.

time is sufficient to differentiate samples. Considering the results of the specificity (measurement of other samples), CZE specifically identified mAbs/ADCs from their peak profiles and their migration times. Therefore, the method shows a high precision and can be used as an identity test for biopharmaceuticals. 


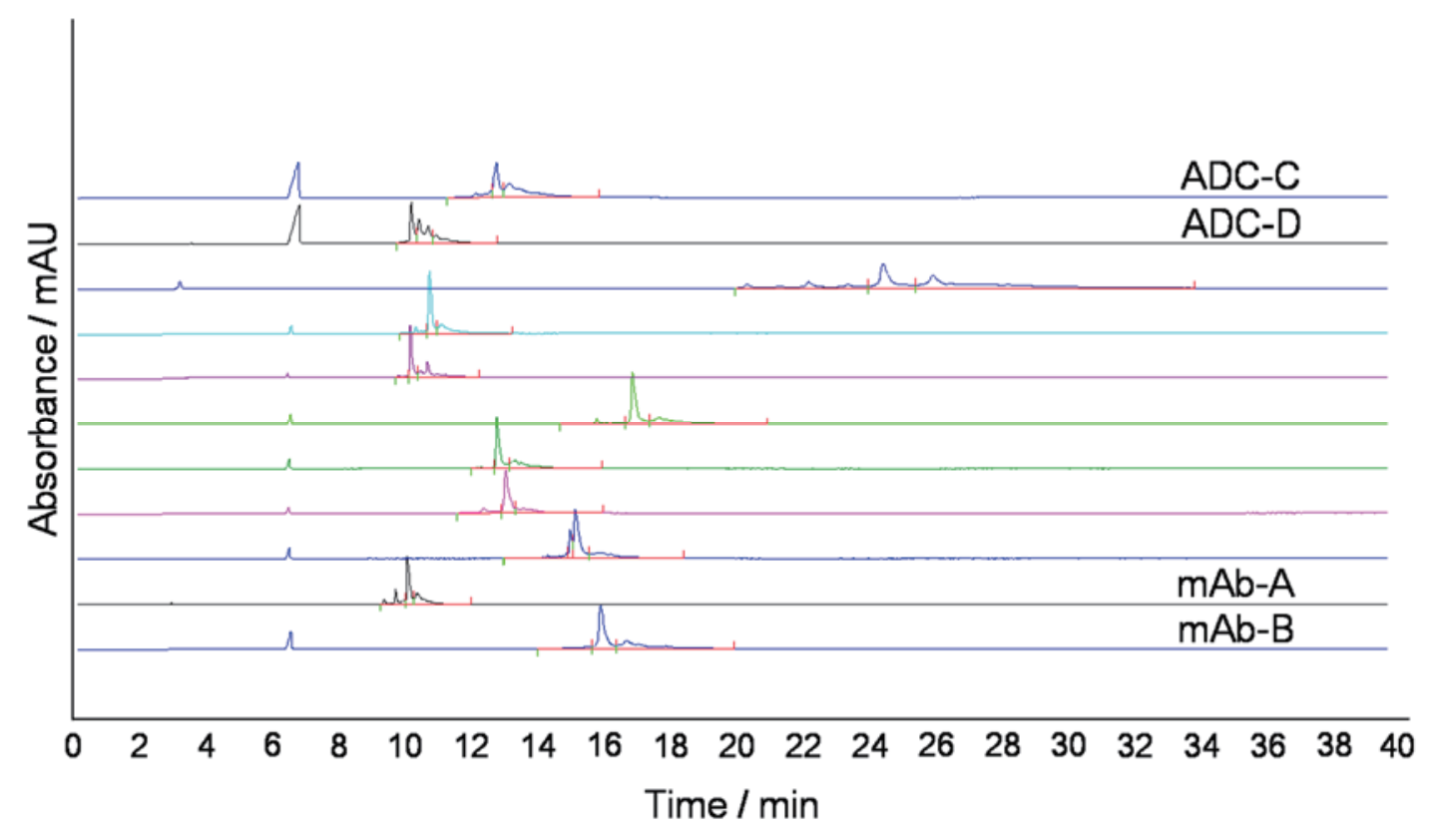

Fig. 4. Electropherograms of the $m A b-A, m A b-B, A D C-C, A D C-D$ and other $m A b$ samples. In total, 11 samples $(2$ ADCs, 9 $\mathrm{mAbs}$ including $\mathrm{mAb}-\mathrm{A}, \mathrm{mAb}-\mathrm{B}, \mathrm{ADC}-\mathrm{C}$, and ADC-D) were analyzed with the same experimental conditions as in Fig. 2.

(a)

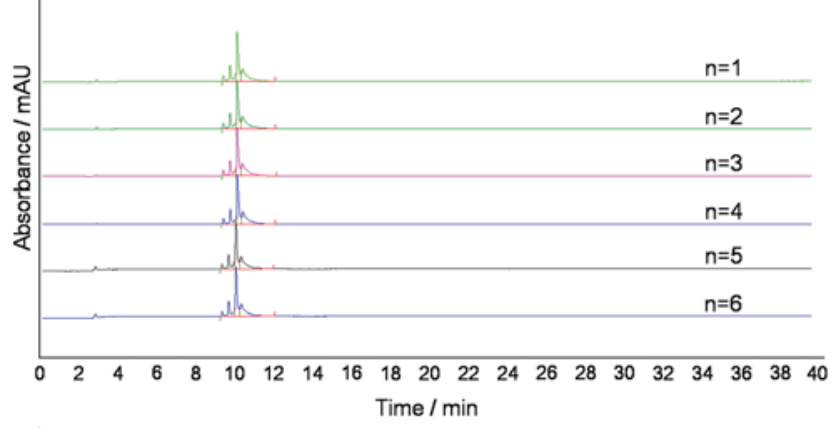

(b)

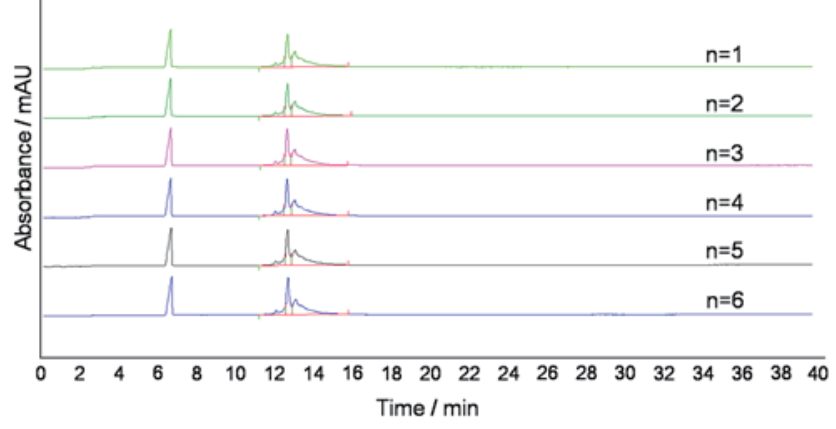

Fig. 5. Repeatability of the (a) mAb-A and (b) ADC-C analysis. Experimental conditions were the same as those in Fig. 2.

\subsection{Accuracy}

Accuracy was evaluated based on the results of the specificity, linearity and precision studies. They showed sufficient analytical performance, therefore, accuracy was confirmed. (a)

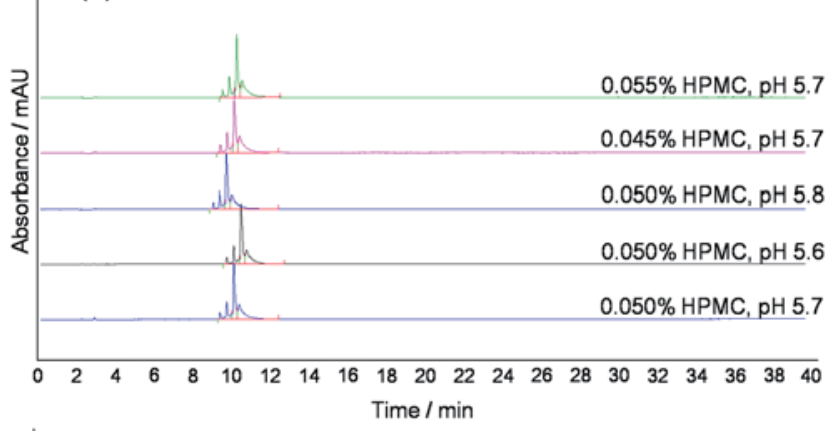

(b)

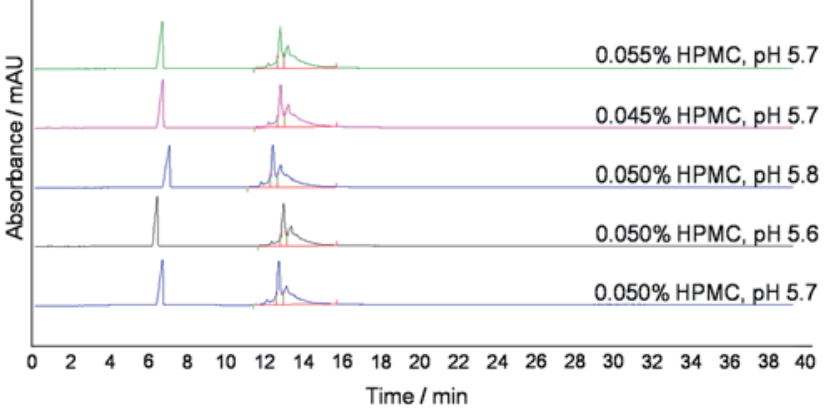

Fig. 6. Effect of $\mathrm{pH}$ and HPMC concentration on the analysis of (a) mAb-A and (b) ADC-C. Experimental conditions were the same as those in Fig. 2.

\subsection{Range}

The results of the specificity, precision and accuracy studies satisfied required analytical performance. Therefore, the range was determined to be a concentration ranging between $1 \%$ to $200 \%$. 


\subsection{Robustness (Running buffer components)}

Fig. 6 shows the effect of running buffer $\mathrm{pH}$ and HPMC concentrations on the electropherograms of mAb-A and ADC-C. The difference (\%) of the main peak area was less than 9.0\%. Peak area did not show any notable change in the range from $0.45 \%$ to $0.55 \%$ HPMC concentration at $\mathrm{pH} 5.7$, or in the range from $\mathrm{pH} 5.6$ to $\mathrm{pH}$ 5.8 with $0.50 \%$ HPMC. Therefore, the method was robust against the changes in $\mathrm{pHs}$ and HPMC concentrations of the running buffer.

\section{Conclusions}

We developed and validated the CZE method to evaluate charge variants of mAbs and ADCs (ranging in $\mathrm{p} I$ from 7 to 9 and having DARs of up to 8). The method validation of CZE was conducted using two different mAbs and two different ADCs. The method was validated for use with identity and purity tests, and thus, can be a promising alternative to the IEF and CEX methods. The method showed quantitative results with high specificity, separation efficiency and precision. It should be noted that CZE is applicable for ADCs without any modification of the method. Therefore, the proposed CZE method shows the potential for the use in manufacturing process development, formulation development, and product characterization of biopharmaceuticals, including ADCs.

\section{References}

[1] Reichert, J. M. mAbs 2012, 4, 413-415.

[2] Walsh, G. Nat. Biotechnol. 2014, 32, 992-1000.

[3] Aggarwal, R. Nat. Biotechnol. 2014, 32, 32-39.

[4] Elvin, J. G.; Couston, R. G.; van der Walle, C. F. Int. J. Pharm. 2013, 440, 83-98.

[5] Ecker, D. M.; Jones, S. D.; Levine, H. L. mAbs 2015, 7, 9-14.

[6] Flygare, J. A.; Pillow, T. H.; Aristoff, P. Chem. Biol. Drug. Des. 2013, 81, 113-121.

[7] Roberts, S. A.; Andrews, P. A.; Blanset, D.; Flagella, K. M.; Gorovits, B.; Lynch, C. M.; Martin, P. L.; Kraamer-Stickland, K.; Thibault, S.; Waner, G. Regul. Toxicol. Pharmacol. 2013, 67, 382-391.

[8] Perez, H. L.; Cardarelli, P. M.; Deshpande, S.; Gangwar, S.; Schroeder, G. M.; Vite, G. D.; Borzilleri, R. M. Drug Discov. Today 2014, 19, 869-881.

[9] Panowski, S.; Bhakta, S.; Raab, H.; Polakis, P.; Junutula, J. R. mAbs 2014, 6, 34-45.

[10] Chari, R. V. J.; Miller, M. L.; Widdison, W. C. Angew. Chem., Int. Ed. 2014, 53, 3796-3827.

[11] Weiner, L. M.; Surana, R.; Wang, S. Nat. Rev. Immunol. 2010, 10, 317-327.

[12] Beck, A.; Reichert, J. M. mAbs 2014, 6, 15-17.

[13] Sassoon, I.; Blanc, V. Methods Mol. Biol. 2013, 1045, $1-27$.

[14] Lin, J.; Tan, Q.; Wang, S. J. Sep. Sci. 2011, 34,
1696-1702.

[15] Kaschak, T.; Boyd, D.; Lu, F.; Derfus, G.; Kluck, B.; Nogal, B.; Emery, C.; Summers, C.; Zheng, K.; Bayer, R.; Amanullah, A.; Yan, B. mAbs 2011, 3, 577-583.

[16] Vlasak, J.; Bussat, M. C.; Wang, S.; Wagner-Rousset, E.; Schaefer, M.; Klinguer-Hamour, C.; Kirchmeier, M.; Corvaia, N.; Ionescu, R.; Beck, A. Anal. Biochem. 2009, 392, 145-154.

[17] Huang, L.; Lu, J.; Wroblewski, V. J.; Beals, J. M.; Riggin, R. M. Anal. Chem. 2005, 77, 1432-1439.

[18] Zhang, J.; Zhang, T.; Jiang, L.; Hewitt, D.; Huang, Y.; Kao, Y. H.; Katta, V. Anal. Chem. 2012, 84, 2313-2320.

[19] Zhang, B.; Yang, Y.; Yuk, I.; Pai, R.; McKay, P.; Eigenbrot, C.; Dennis, M.; Katta, V.; Francissen, K. C. Anal. Chem. 2008, 80, 2379-2390.

[20] Liu, Y. D.; Goetze, A. M.; Bass, R. B.; Flynn, G. C. J. Biol. Chem. 2011, 286, 11211-11217.

[21] Chelius, D.; Jing, K.; Lueras, A.; Rehder, D. S.; Dillon, T. M.; Vizel, A. ; Rajan, R. S.; Li, T.; Trsuheit, M. J.; Bondarenko, P. V. Anal. Chem. 2006, 78, 2370-2376.

[22] Khawli, L.; Goswami, S.; Hutchinson, R.; Kwong, Z.; Yang, J.; Wang, H.; Yao, Z.; Sreedhara, A.; Cano, T.; Tesar, D.; Nijem, I.; Allison, D.; Wong, P.; Kao, Y.; Quan, C.; Joshi, A.; Harris, R.; Motchnik, P. mAbs 2010, 2, 613-624.

[23] Chaderjian, W. B.; Chin, E. T.; Harris, R. J.; Etcheverry, T. M. Biotechnol. Prog. 2005, 21, 550-553.

[24] Zhang, L.; Patapoff, T.; Farnan, D.; Zhang, B. J. Chromatogr. A 2013, 1272, 56-64.

[25] Fekete, S.; Beck, A.; Fekete, J.; Guillarme, D. J. J. Pharm. Biomed. Anal. 2015, 102, 33-44.

[26] Fekete, S.; Beck, A.; Fekete, J.; Guillarme, D. J. J. Pharm. Biomed. Anal. 2015, 102, 282-289.

[27] Talebi, M.; Shellie, R. A.; Hilder, E. F. Anal. Chem. 2014, 86, 9794-9799.

[28] Talebi, M.; Nordborg, A.; Gasper, A.; Lacher, N. A.; Wang, Q.; He, X. Z.; Haddad, P. R.; Hilder, E. F. J. Chromatogr. A 2013, 1317, 148-154.

[29] Kang, X.; Kutzko, J. P.; Hayes, M. L.; Frey, D. D. J. Chromatogr. A 2013, 1283, 89-97.

[30] Rea, J. C.; Moreno, T.; Lou, Y.; Farnan, D. J. J. Pharm. Biomed. Anal. 2011, 54, 317-323.

[31] Wenisch, E.; Reiter, S.; Hinger, S.; Steindl, F.; Tauer, C.; Jungbauer, A.; Katinger, H.; Righetti, P. G. Electrophoresis 1990, 11, 966-969.

[32] Mack, S.; Cruzado-Park, I.; Chapman, J.; Ratnayake, C.; Vigh, G. Electrophoresis 2009, 30, 4049-4058.

[33] Salas-Solano, O.; Kennel, B.; Park, S. S.; Zhang, X.; Zhang, L.; Sosic, Z.; Boumajny, B.; Zeng, M.; Cheng, K. C.; Reed-Bogan, A.; Cummins-Bitz, S.; Michels, D. A.; Parker, M.; Bonasia, P.; Hong, M.; Cook, S.; 
Ruesch, M.; Lamb, D.; Bolyan, D.; Kiessig, S.; Allender, D.; Nunnally, B. Chromatographia 2011, 73, 1137-1144.

[34] Suba, D.; Urbanyi, Z.; Salgo, A. J. Pharm. Biomed, Anal. 2015, 114, 53-61.

[35] He, X.; Que, A.; Mo, J. Electrophoresis 2009, 30, 714-722.

[36] Zhang, J.; Yip, H.; Katta, V. Anal. Biochem. 2011, 410, 234-243.

[37] Sosic, Z.; Houde, D.; Blum, A.; Carlage, T.; Lyubarskaya, Y. Electrophoresis 2008, 29, 4368-4376.

[38] Salas-Solano, O.; Kennel, B.; Park, S. S.; Roby, K.; Sosic, Z.; Boumajny, B.; Free, S.; Reed-Bogan, A.; Michales, D.; McElroy, W.; Bonasia, P.; Hong, M.; He, X.; Ruesch, M.; Moffatt, F.; Kiessig, S.; Nunnally, B. J. Sep. Sci. 2012, 35, 3124-3129.

[39] He, Y.; Lacher, N. A.; Hou, W.; Wang, Q.; Isele, C.; Starkey, J.; Ruesch, M. Anal. Chem. 2010, 82, 3222-3230.

[40] He, Y.; Isele, C.; Hou, W.; Ruesch, M. J. Sep. Sci. 2011, 34, 548-555.

[41] Shi, Y.; Li, Z.; Qiao, Y.; Lin, J. J. Chromatogr. B 2012, 906, 63-68.

[42] Espinosa-de la Garza, C. E.; Perdomo-Abundez, F. C.; Padilla-Calderon, J.; Uribe-Wiechers, J. M.; Perez, N. O.; Flores-Ortiz, L. F.; Medina-Rivero, E. Electrophoresis 2013, 34, 1133-1140.

[43] Han, H. L.; Livingston, E.; Chen, X. Y. Anal. Chem. 2011, 83, 8184-8191.

[44] Li, S. K.; Liddell, M. R.; Wen, H. J. Pharm. Biomed. Anal. 2011, 55, 603-607.

[45] Zhao, S. S.; Chen, D. D. Y. Electrophoresis 2014, 35, 96-108.

[46] Biacchi, M.; Gahoual, R.; Said, N.; Beck, A.; Leize-Wagner, E.; François, Y. N. Anal. Chem. 2015, 87, 6240-6250.

[47] Moritz, B.; Schnaible, V.; Kiessig, S.; Heyne, A.; Wild, M.; Finkler, C.; Christians, S.; Mueller, K.; Zhang, L.; Furuya, K.; Hassel, M.; Hamm, M.; Rustandi, R.; He, Y.; Salas-Solano, O.; Whitmore, C.; Ae Park, S.; Hansen, D.; Santos, M.; Lies, M. J. Chromatogr. B: Anal. Technol. Biomed. Life Sci. 2015, 983, 101-110.

[48] Michels, D. A.; Salas-Solano, O.; Felten, C. BioProcess Int. 2011, 9, 48-54. 